\title{
Materiales compuestos de matriz metálica. Il parte. Métodos de procesado y consolidación de MMCs reforzados con partículas $\left(^{(*)}\right.$
}

\author{
Cesar Edil da Costa*, Francisco Velasco López ${ }^{* *}$ y Jose Manuel Torralba Castelló**
}
Resumen En este trabajo se ofrece una revisión de los distintos métodos de conformado y consolidación de materiales compuestos de matriz metálica reforzados con partículas. En una primera parte se hace hincapié en los métodos de conformado a partir de las materias primas y en la segunda en métodos de consolidación final a partir de preformas.

Palabras clave Materiales compuestos de matriz metálica reforzados con partículas. Squeeze casting. Reocolada. Spray forming. Pulvimetalurgia. Extrusión. Laminación.

\section{Metal matrix composites. Part 2. Processing and consolidation techniques for particle reinforced MMCs}

\begin{abstract}
This work is a review of the different forming and consolidation methods for particulate metal matrix composites. In the first part we focus in the forming methods from raw materials while the second is centred in the consolidation from preforms in order to get the final part.
\end{abstract}

Keywords Particulate metal matrix composites. Squeeze casting. Rheocasting. Spray forming. Powder metallurgy. Extrusion. Rolling.

\section{INTRODUCCIÓN}

Normalmente, los materiales compuestos de matriz metálica (MMCs) reforzados con partículas necesitan dos etapas para obtener una pieza con forma y propiedades finales. En una primera fase de procesado se obtiene una preforma a partir de las materias primas y, posteriormente, se pasa a una et de consolidación final donde el material adquiere todas sus prestaciones. El objetivo de este trabajo es revisar los principales métodos de procesado y consolidación de estos materiales.

\section{MÉTODOS DE PROCESADO}

Los principales métodos de procesado de materiales compuestos de matriz metálica son:

- Squeeze casting.

- Reofusión o reocolada
- Deposición por spray (spray forming).

- Pulvimetalurgia.

En los siguientes apartados se detallan cada uno de ellos.

\subsection{Squeeze casting ${ }^{[1]}$}

En este método de fabricación de MMCs, los refuerzos más utilizados son whiskers de $\mathrm{SiC}$ y en general fibras cortas. Inicialmente, se fabrica una preforma del material cerámico usando, normalmente, un aleante. Esta preforma se coloca en una matriz. A continuación se adiciona el metal líquido y, seguidamente, por aplicación de presión, éste se infiltra en la preforma (Fig. 1).

Para obtener un producto de buena calidad es necesario un control cuidadoso de la temperatura de la preforma, el ciclo de presión, la temperatura del metal líquido y las condiciones de

\footnotetext{
(•) Trabajo recibido el día 23 de septiembre de 1999 y aceptado en su forma final el 5 de abril de 2000.

(*) Universidade do Estado de Santa Catarina. Dept. Ing. Mecánica. Campus Universitario Bom Retiro, $89223-100$ Joinville /SC (Brasil).

(**) Universidad Carlos III de Madrid. Dpto. Materiales. Avda. Universidad 30.28911 Leganés (Madrid, España).
} 


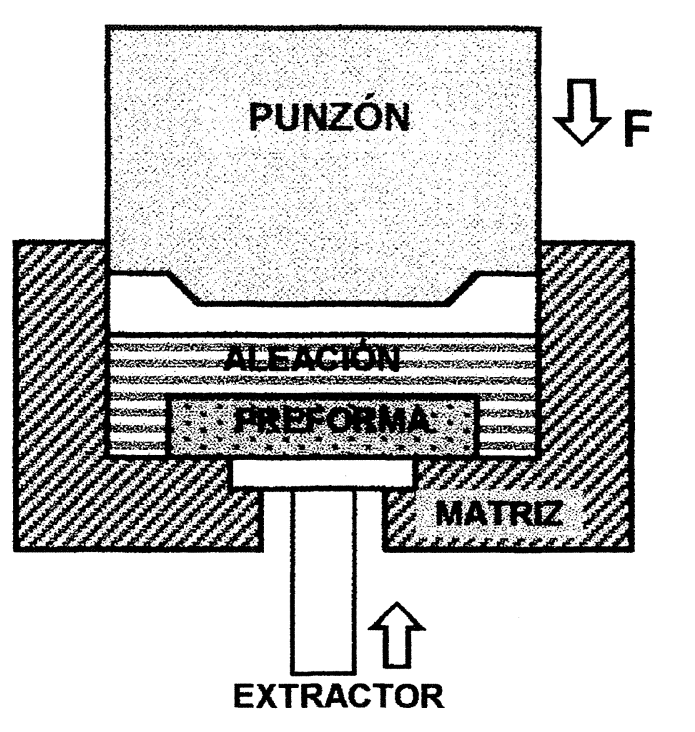

Figura 1. Proceso de obtención de materiales compuestos por "squeeze casting".

Figure 1. Squeeze casting of metal matrix composites.

solidificación, La temperatura de la preforma y la del metal líquido influyen en la presión requerida para la infiltración del metal. Además, la aplicación de presión también produce una compresión de la preforma y si esta presión es muy elevada puede provocar la fractura de la misma y consecuentemente de las fibras.

Se deben controlar las condiciones de solidificación para evitar el encuentro de los frentes de solidificación y la aparición de porosidad.

Por medio de este proceso se puede infiltrar, también, una preforma en una pieza, en la que se desea reforzar una determinada zona. Este procedimiento se utiliza para producir piezas como pistones de motores de combustión interna, donde se desea reforzar, solamente, las regiones más solicitadas.

\subsection{Reofusión o reocolada ${ }^{[1]}$}

Consiste en el conformado de un material en estado parcialmente solidificado, es decir, donde las cantidades de fase líquida y sólida están delimitadas por las líneas de líquidus y sólidus del diagrama de fases (Fig. 2). Este proceso presenta dificultades relacionadas con el carácter dendrítico de la solidificación, que provoca la imposibilidad de verter fácilmente la aleación en un molde cuando la fracción solidificada sea entre 20-30\%, aproximadamente. En este caso el material no es capaz de llenar el molde sin que aparezcan grietas ni se segreguen fases líquidas.

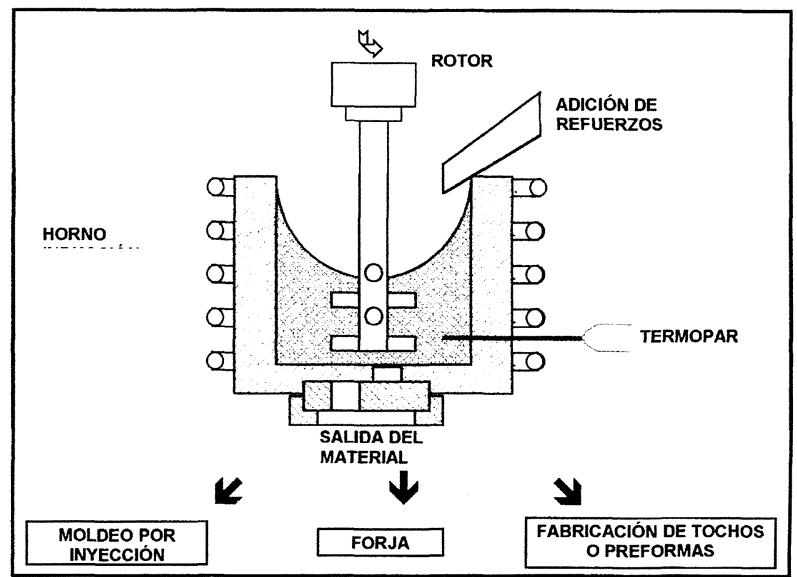

Figura 2. Proceso de fabricación de materiales compuestos por reofusión.

Figure 2. Rheocasting of metal matrix composites.

La introducción de un elevado esfuerzo de cizalla (tipo agitación mecánica) en el metal líquido, duránte la solidificación, impide la formación de esta estructura dendrítica, promoviendo la aparición de una estructura esferoidal.

El mayor problema en la fabricación de MMCs por medio de reofusión es conseguir un mojado suficiente entre el baño metálico y el refuerzo. Debido a que el tiempo de contacto entre la partícula de refuerzo y el metal líquido puede ser muy largo, la posible reacción entre las dos fases es de gran importancia. El refuerzo elegido debe ser estable en contacto con el metal líquido o, en caso contrario, debe ser tratado para proporcionar una capa protectora. La distribución de las partículas depende, fundamentalmente, del método de mezcla y de la velocidad de solidificación.

\subsection{Deposición por spray (spray forming) ${ }^{[2]}$}

Este proceso está basado en la técnica de producción de polvos vía atomización por gas, que consiste en la atomización de un chorro de metal líquido por la acción de un haz de gas inerte. En este caso, sin embargo, las partículas de metal líquido producidas por atomización, chocan con un substrato intermedio, constituyendo una preforma. Este substrato es una matriz con la forma deseada (Fig. 3).

La fabricación de MMCs por este método se realiza mediante la introducción de partículas de refuerzo dentro del chorro de atomización para ser codepositadas e incorporadas a la aleación solidificada. El tiempo de contacto entre el metal líquido y las partículas de refuerzo es muy corto. Este hecho, unido a que las velocidades de solidificación 


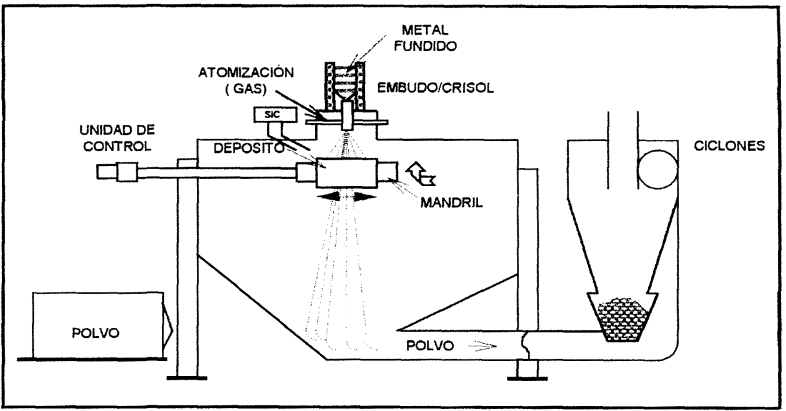

Figura 3. Proceso de obtención de materiales compuestos por "spray".

Figure 3. Spray forming of metal matrix composites.

provocadas por el impacto de las partículas con el substrato son elevadas, reduce considerablemente la posibilidad de reacciones interfaciales. Se minimiza así la formación de compuestos interfaciales frágiles, como el $\mathrm{Al}_{4} \mathrm{C}_{3}$, cuando se refuerza aluminio con grafito, que pudieran afectar las buenas propiedades del material.

Normalmente el flujo de metal es de $5 \mathrm{~kg} / \mathrm{min}$ y la preforma obtenida presenta una densidad aproximada del $95 \%$. A continuación, tiene lugar una etapa secundaria de procesado, como extrusión, laminado o forja para propiciar la densidad total del material.

Este método de producción de compuestos confiere a las piezas una microestructura muy fina, con una distribución homogénea de los refuerzos, y mantiene una mayor cantidad de elementos de aleación en solución.

\subsection{Pulvimetalurgia (P/M)}

El proceso de obtención de MMCs por vía pulvimetalúrgica ${ }^{[3-6]}$ consiste, básicamente, en la mezcla de polvos metálicos y refuerzos, en estado sólido, seguida de compactacion y consolidación. Este proceso presenta la ventaja de requerir menores temperaturas durante la preparación de los compuestos, en comparación con la obtención de compuestos en estado líquido. De esta forma, la interacción entre matriz y refuerzo es menor por lo que se minimizan las reacciones interfaciales indeseables $\left(\mathrm{Mg}+\mathrm{Al}_{2} \mathrm{O}_{3}, \mathrm{Al}+\mathrm{SiC}, \mathrm{T}+\mathrm{C}\right.$, etc. $)$, posibilitando, de esta forma, una mejora de las propiedades mecánicas. La figura 4 presenta las principales etapas del procesado por vía pulvimetalúrgica.

Es de gran importancia que las partículas de los polvos metálicos y refuerzos estén uniformemente distribuidas en la mezcla, a fin de obtener una estructura homogénea. En el caso de MMCs reforza-

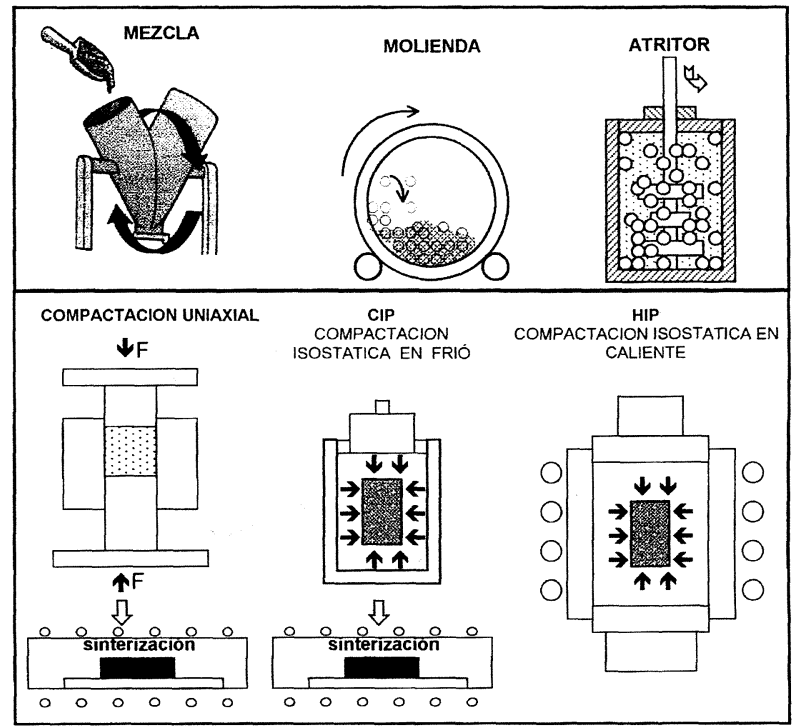

Figura 4. Proceso de fabricación de materiales vía pulvimetalúrgica.

Figure 4. Powder metallurgy process.

dos con whiskers se requieren polvos metálicos muy finos, para que mejoren el empaquetamiento y produzcan una dispersión uniforme.

Las propiedades de los MMCs fabricados por vía pulvimetalúrgica pueden ser mejoradas a través de sinterización con fase liquida, con o sin presión adicional, y también por procesos de fabricación secundarios tales como extrusión, laminación y forja.

\section{CONSOLIDACIÓN DE LOS MATERIALES COM- PUESTOS DE MATRIZ METÁLICA: EXTRUSIÓN}

\subsection{Introducción}

La extrusión es un proceso ${ }^{[7-13]}$ por el cual se pueden obtener formas o perfiles, como tubos sin costura o alambres, que difícilmente se obtienen por medio de otros procesos. Por estas características, es el proceso preferido en la fabricación de MMCs. La figura 5 muestra la secuencia de varias posibilidades de extrusión a partir de una preforma pulvimetalúrgica.

\subsection{Temperatura de trabajo}

La temperatura de trabajo, es decir, la temperatura de extrusión, debe ser lo suficientemente alta para conferir elevada conformabilidad plástica al metal. A pesar de que el límite superior se puede fijar alrededor de $50{ }^{\circ} \mathrm{C}$ por debajo de la temperatura de fusión del metal (o aleación), en la realidad es menor, por la posibilidad de que aparezcan fenómenos de fragilidad en caliente, provocados por la 


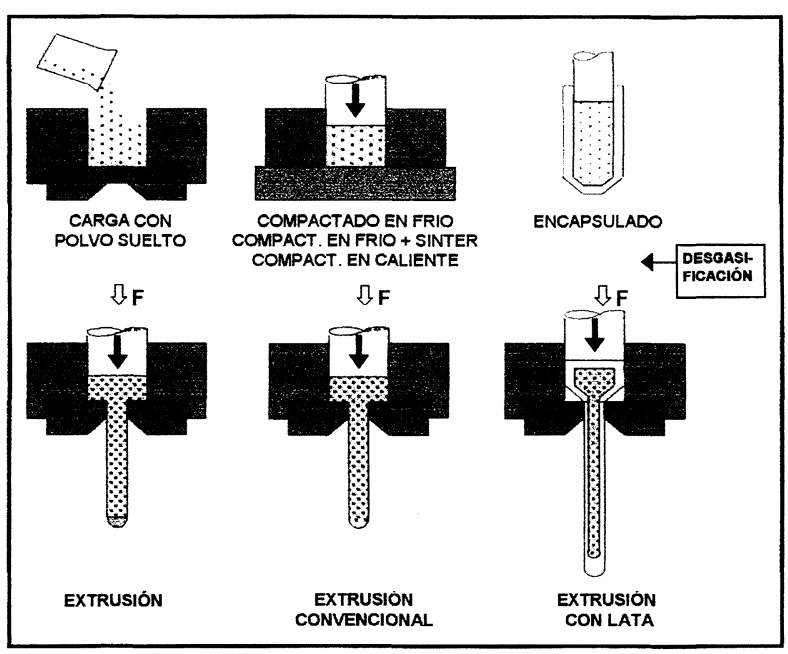

Figura 5. Procesos de extrusión de materiales pulvimetalúrgicos.

\section{Figure 5. Extrusion process from $P / M$ preforms.}

fusión de microconstituyentes de menor punto de fusión. A veces estos fenómenos van asociados a las impurezas que conducen a la desagregacion por fisuramiento de la preforma por deformación.

Por otro lado, la temperatura no debe ser muy elevada para evitar un consumo excesivo de energía, el desgaste de los elementos de las máquinas de extrusión y la oxidación excesiva de la preforma.

Para materiales en los que la temperatura de conformado es baja, se puede calentar la preforma y la matriz a la misma temperatura (conformado isotérmico), evitando así pérdidas excesivas de calor y, en consecuencia, el desgaste de la herramienta.

\subsection{Velocidad de extrusión}

Las velocidades usuales de trabajo en la extrusión pueden ser diversas. Por un lado, se busca utilizar niveles altos para conferir mayor producción a las máquinas de extrusión. Sin embargo los niveles de presión de extrusión, para una determinada temperatura de trabajo, se elevan con el aumento de la velocidad.

La menor pérdida de temperatura, con mayores velocidades, evita que se produzca una fuerte elevación de la presión de extrusión. Con la matriz de extrusión calentada a temperaturas más altas, la influencia de la velocidad en la presión de extrusión se reduce.

\subsection{Lubricación}

El desgaste entre la matriz y la preforma y, entre la herramienta y la preforma bajo extrusión, tiene un acentuado efecto en la fluencia del material con repercusión en la calidad del producto obtenido en la extrusión, bien en términos de propiedades mecánicas, bien en cuanto a la presencia o no de defectos de extrusión.

Los aceites con grafito, o solamente el grafito, resistentes a elevadas temperaturas, se aplican a la matriz y a la preforma en los casos en que se pretenda realizar la extrusión con lubricante, mejorando así el acabado superficial del producto extruído y reduciendo el efecto de desgaste en la herramienta. Para algunas aleaciones metálicas, no se utilizan lubricantes pues pueden manchar la superficie del producto extruído.

\subsection{Geometría de la herramienta}

No hay una regla exacta para relacionar el ángulo de entrada (ángulo del cono) y el radio de acuerdo en la punta de la herramienta al nivel de presión de extrusión pues, a medida que la presión tiende a caer con un radio de acuerdo mayor o un ángulo de cono mayor, el aumento del área de contacto con el material aumenta esta presión con el consiguiente aumento de la fuerza de fricción. Conviene resaltar que el aumento de la parte cilíndrica de la herramienta, usada para regularizar y calibrar las dimensiones de la sección transversal del extruído, también aumenta la fuerza de extrusión por desgaste. Muchas veces se usan herramientas de superficies fijas, esto es, con ángulos de $180^{\circ}$ y con pequeños radios de acuerdo. Una herramienta de este tipo presenta, sin embargo, un flujo de material menos uniforme que las que tienen apertura cónica, a pesar de que estas ultimas presentasen un mayor nivel de fuerza de rozamiento.

\subsection{Presión de extrusión}

La presión de extrusión depende de diversos factores, tales como la naturaleza del material, su cambio con la temperatura, con la velocidad de trabajo y la geometría de la herramienta. Además de esto, depende también de la reducción (cuanto mayor reducción, mayor presión) y de la forma del perfil extruído. La principal dificultad en el establecimiento de las relaciones entre los factores de influencia y la presión es el efecto de interacción de estos factores. Obsérvese sin embargo, que el factor de influencia más importante es la intensidad de reducción en área, que acentúa notablemente el crecimiento de la presión a partir de niveles del 90 $\%$, para un determinado material, a niveles de temperatura y velocidad constantes. 


\subsection{Otros procesos de consolidación}

La laminación y la forja ${ }^{[14-20]}$ son dos procesos secundarios importantes en la fabricación de productos a partir de materiales compuestos. Tienen como objetivo mejorar la estructura final y aumentar las propiedades por efecto de la deformación, así como la eliminación de porosidad.

El proceso de forja es el más adecuado para la obtención de materiales en forma próxima al producto final (near-net-shape), caracterizándose por ser un proceso de producción de piezas con mucha precisión dimensional. El proceso de laminado nos permite obtener materiales compuestos del tipo sándwich, o materiales con recubrimientos a partir de polvos.

\section{CONCLUSIÓN}

Como se puede ver en esta revisión, podemos elegir entre muy distintos métodos de procesado para fabricar materiales compuestos de matriz metálica reforzados con partículas. Cada uno de estos métodos permite obtener propiedades y características específicas del material final (vinculadas de forma inherente al proceso de fabricación), incluso para las mismas composiciones químicas y mismo porcentaje de refuerzo. Pese a que puede haber grandes diferencias económicas entre los distintos métodos de procesado, podemos encontrar entre ellos métodos de procesado de coste medio-bajo, por lo que estos materiales se pueden utilizar en procesos de fabricación para grandes series.

\section{REFERENCIAS}

[1] D.J. LLOYD, D.J. Adv. Struct. Mater. (1990) 1-21.
[2] S.J. HARRIS, AGARD Lectures Senes $n^{\circ}$ 174: New Light Alloys, 1990: 4:1-4:21.

[3] "Powder Metallurgy" - Metals Handbook, ASM, Materials Park, vol. 7, 9으. Ed., 1984.

[4] W.J. UlLRICH, Prog. Powder Metall. 46 (1986) 535-556.

[5] D.P. Voss, D.P. Mod. Dev. Powder Metall. 13 (1981) 467-481.

[6] D.L. ERICH, Prog. Powder Metall. 46 (1986) 45-65.

[7] W.T. Nachtrab y P.R. Roberts, Adv. Powder Metall. Particulate Mater. 4 (1992) 321-335.

[8] R. DASGUPTA Y S.K. BOSE, J. Mater. 47 (1995) 20-22.

[9] J. Grosch y H. Janiche, H. Mod. Dev. Powder Metall. 10 (1977) 325-346.

[10] V.V. ZAKHAROV, V.V. Adv. Performance Mater. 2 (1995) 51-56.

[11] A.R. Austen, W.L. Hutchinson y W.H. Fellbach. Met. Powder Rep. 41 (1980) 294-298.

[12] A. Jokinen, A. Mod. Dev. Powder Metall. 19 (1988) 547-563.

[13] J.H. Weber y A.I. KempPInen, Adv. Powder Metall. Particulate Mater. 7 (1992) 303-314.

[14] Y. WANG y G.W. Lorimer, Proc. of the World Congress on Powder Metallurgy, SF2M, vol. III, Paris, Francia, 1994, pp. 1635-1638.

[15] H. COHRT, Adv. Powder Metall. Particulate Mater. 9 (1992) 33-43.

[16] H. Morimoto y K-I. OhuchI, K-I. Met. Powder Rep. 45 (1990) 706-708.

[17] K.M. KulKarnI y S. BhattacharYya, Powder Metall. Defence Technol. 4 (1978) 49-65.

[18] G. JANGG, G. Met. Powder Rep. 35 (1980) 206-208.

[19] X. NI, M.S. MAClean y T.N. Baker, Proc. of the World Congress on Powder Metallurgy, SF2M, vol. III, Paris, Francia, 1994 pp. 1983-1986.

[20] S. Chekroud, B. Munar y G. Cizeron, Colloquium: Controlling the Properties of Powder Metallurgy Parts Through their Microstructure, SF2M, Paris, Francia, 1990, 20.1-20.6. 